\title{
Un índice neutrófilo/linfocito elevado se asocia a peor pronóstico en cáncer de colon etapa II resecado
}

\author{
GONZALO I. URREJOLA ${ }^{1}$, CLAUDIA E. BAMBS ${ }^{1}$, \\ MANUEL A. ESPINOZA², JOSÉ GELLONA ${ }^{1}$, ÁLVARO M. ZÚÑIGA ${ }^{1}$, \\ MARÍA ELENA MOLINA ${ }^{1}$, FELIPE BELLOLIO ${ }^{1}$, RODRIGO MIGUIELES ${ }^{1}$, \\ JAMES M. CAMPBELL ${ }^{1}$, GEORGE A. PINEDO ${ }^{1 \dagger}$
}

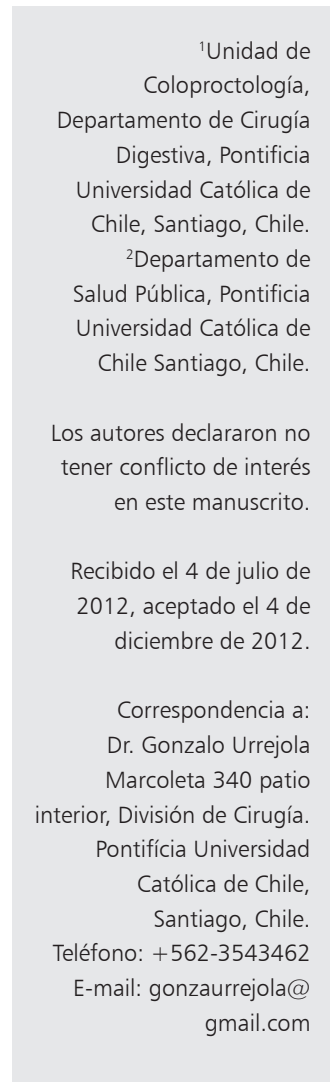

\section{An elevated neutrophil/lymphocyte ratio is associated with poor prognosis in stage II resected colon cancer}

Background: The neutrophil/lymphocyte ratio is an effective marker of inflammation and can have prognostic value in surgical patients. Aim: To evaluate the effect of an increased neutrophil/lymphocyte ratio (NLR) on perioperative complications and overall and disease-free survival in patients undergoing elective resection for stage II colon cancer. Material and Methods: Data was obtained from clinical charts, preoperative blood results and hospital records of all patients undergoing an elective curative resection for colon cancer, between 2000 and 2007. Preoperative NLR was calculated. Follow-up was obtained from a prospectively maintained colorectal cancer database, clinical records and questionnaires. Uni and multivariable analysis were performed to identify associations, and survival analysis was performed using KaplanMeier curves. Results: One hundred twenty two patients with a mean age of 69 years (52\% males), were evaluated. Median follow-up was 73 months, and overall survival for 1 and 5 years was 95\% and 68\%, respectively. On a multivariable analysis after adjusting for age, sex, tumor depth invasion, use of adjuvant therapies and American Society of Anesthesiology preoperative risk score, an NLR $>5$ was associated with an increased perioperative complication rate (odds ratio: 3,06, $p=0,033$ ). Kaplan-Meier survival analysis showed a worse overall and disease-free survival for patients with NLR greater than five. Conclusions: A preoperative NLR of five or more is associated with greater perioperative morbidity and worse oncological outcomes in patients undergoing resection for elective stage II colon cancer.

(Rev Med Chile 2013; 141: 602-608).

Key words: Colonic neoplasms; Lymphocytes; Neutrophils.
E l cáncer colorrectal (CCR) continúa siendo una de las neoplasias más frecuentes en todo el mundo ${ }^{1,2}$. A pesar de los avances en la técnica quirúrgica, cuidados peri operatorios y terapia adyuvante, sólo $50 \%$ de los pacientes está vivo a los 5 años después de una cirugía potencialmente curativa $^{3}$. En pacientes etapa II de la AJCC, es decir, tumores T3 o T4 sin linfonodos comprometidos, la quimioterapia adyuvante se considera cuando existen factores clínicos o histopatológicos de peor pronóstico $^{4-6}$. En los últimos años, se han tratado de identificar nuevos marcadores histopatológicos ${ }^{7}$ y molelulares ${ }^{8}$.

La inflamación y sus efectos en la carcinogénesis han motivado interés y extensa investigación. Últimamente la atención se ha puesto en la inflamación sistémica inducida por los tumores. La concomitante elevación de citoquinas y otros 
mediadores inflamatorios inhibiría la apoptosis tumoral, promovería la angiogénesis y dañaría el $\mathrm{ADN}^{9-12}$. Todos estos factores contribuirían a una mayor probabilidad de recidiva local o sistémica.

El índice neutrófilo/linfocito (NLR), definido como el recuento absoluto de neutrófilos dividido por el recuento de linfocitos, es un marcador efectivo de inflamación que se está usando cada vez más para evaluar resultados en pacientes quirúrgicos; su utilidad ha sido demostrado en pacientes críticos $^{13,14}$ y algunas enfermedades neoplásicas ${ }^{15,16}$. Walsh et $\mathrm{al}^{17}$ estudiaron el valor pronóstico de un NLR $\geq 5$ en CCR, y lo identificaron como un marcador de sobrevida. Sin embargo, todas las etapas oncológicas del cáncer fueron evaluadas en conjunto, y se incluyeron casos de cirugía de urgencia. El NLR apareció asociado a la etapa oncológica en este estudio, pero no se logró demostrar como un factor de riesgo independiente de la etapificación de Duke. Halazun et $\mathrm{al}^{15}$ demostraron que un NLR elevado fue predictor de recurrencia y peor sobrevida en pacientes con metástasis hepáticas de CCR. Ambas publicaciones coinciden en un valor de corte de 5 para el NLR.

El objetivo de este estudio es evaluar el valor pronóstico de un NLR elevado en pacientes que van a cirugía curativa electiva en cáncer de colon en etapa II. Se plantea la hipótesis de que un NLR $\geq 5$ predice mayor morbilidad peri operatoria, $y$ peor sobrevida global y libre de enfermedad.

\section{Pacientes y Método}

Se incluyeron todos los pacientes con cáncer de colon demostrado por histología, etapa TNM II, que fueron sometidos a cirugía curativa, en la unidad de coloproctología del Hospital Clínico de la Pontificia Universidad Católica de Chile, entre enero de 2000 y enero de 2007. Se excluyeron pacientes con enfermedades hematológicas, infección activa al momento de la cirugía y aquellos que requirieron cirugía de urgencia por obstrucción o peritonitis. Un total de 409 pacientes fueron sometidos a cirugía por cáncer de colon, de los cuales 169 se encontraban en etapa II. Cuarenta y siete presentaron algún criterio de exclusión, por lo que en el estudio se incluyeron 122 pacientes, de los cuales 5 (4\%) se perdieron durante el seguimiento. Este estudio fue aprobado por el comité de ética del hospital.

\section{Recolección de datos}

Los datos fueron recolectados de la base de datos prospectiva de CCR, de la ficha clínica y cuestionarios personalizados. El NLR se consideró la variable independiente y se calculó del hemograma preoperatorio que se toma de rutina previo a la cirugía. Se utilizó como punto de corte un valor de 5. El resultado primario se definió como la morbilidad perioperatoria, estratificada de I a V según la clasificación de Clavien, con ocurrencia dentro de los 30 días del procedimiento quirúrgico. Los resultados secundarios fueron la sobrevida global y libre de enfermedad. Los pacientes fueron seguidos hasta su fallecimiento o cuando se llegó al final del estudio. La edad, sexo, clasificación de ASA, inmunosupresión, tipo de procedimiento quirúrgico, adyuvancia y tumores T4 fueron considerados confundentes para el análisis.

\section{Análisis estadístico}

Las variables discretas fueron comparadas usando $\chi^{2}$ o test de Fisher, mientras que las variables continuas se evaluaron con prueba t. Para evaluar el efecto de los confundentes en la morbilidad perioperatoria se realizó un análisis uni y multivariado con regresión logística. El análisis de sobrevida se estimó usando el método de Kaplan Meier, y las diferencias entre curvas fueron probadas con el test de log-rank. Para el análisis estadístico se utilizó STATA 10, y se consideró un valor $\mathrm{p}<$ a 0,05 como estadísticamente significativo.

\section{Resultados}

Las características basales, tipo de cirugía y detalles anatomopatológicos se exponen en la Tabla 1. El promedio de edad fue de 69 años, $51 \%$ de los pacientes eran de sexo masculino, y $88 \%$ fueron clasificados como ASA 1 ó 2. La cirugía realizada con mayor frecuencia fue la hemicolectomía derecha (46 pacientes). El promedio de seguimiento fue de 73 meses (rango 20-114). Se observaron complicaciones perioperatorias en 27 pacientes $(22 \%)$, de los cuales 8 tuvieron más de una complicación (Tabla 2). Un paciente murió de falla multiorgánica. Veintiún pacientes (17\%) desarrollaron recurrencia y 24 pacientes murieron durante el seguimiento. Catorce de estas muertes fueron debidas al CCR. La sobrevida global a 1 y 5 años fue de $95 \%$ y $68 \%$ respectivamente. El análisis 
Tabla 1. Características basales, extensión de la cirugía y detalles anatomopatológicos de pacientes con cáncer de colon etapa II

\begin{tabular}{|c|c|c|c|c|}
\hline Característica & $\begin{array}{l}\text { NLR }<5 \\
(n=94)\end{array}$ & $\begin{array}{l}\text { NLR }>5 \\
(n=28)\end{array}$ & $\begin{array}{c}\text { Total } \\
\text { (n = 122) }\end{array}$ & $\mathbf{p}$ \\
\hline Edad, años (SD) & $69(12)$ & $70(15)$ & $69(13)$ & $0,83^{*}$ \\
\hline Masculino (\%) & $47(50)$ & $16(57,1)$ & $63(51,6)$ & 0,51 * \\
\hline $\begin{array}{l}\text { Puntaje ASA (\%) } \\
1-2 \\
3-4\end{array}$ & $\begin{array}{r}87(92,6) \\
7(7,4)\end{array}$ & $\begin{array}{r}21(75) \\
7(25)\end{array}$ & $\begin{array}{r}108(88,5) \\
14(11,5)\end{array}$ & $0,018^{* *}$ \\
\hline Inmunosupresióna (\%) & $10(10,6)$ & $4(14,3)$ & $14(11,5)$ & $0,59 *$ \\
\hline $\begin{array}{l}\text { Operación } \\
\text { Hemicolectomía derecha (\%) } \\
\text { Hemicolectomía izquierda (\%) } \\
\text { Sigmoidectomía (\%) } \\
\text { Colectomía subtotal (\%) } \\
\text { Hemicolectomía derecha extendida (\%) } \\
\text { Otras resecciones segmentarias }{ }^{\mathrm{b}}(\%)\end{array}$ & $\begin{array}{c}35(37,2) \\
25(26,6) \\
17(18) \\
6(6,4) \\
4(4,2) \\
8(8,5)\end{array}$ & $\begin{array}{c}11(39,3) \\
7(25) \\
3(10,7) \\
2(7,1) \\
2(7,1) \\
3(10,7)\end{array}$ & $\begin{array}{c}46(37,7) \\
32(26,2) \\
19(15,6) \\
8(6,6) \\
6(4,9) \\
11(9)\end{array}$ & $\begin{array}{l}0,84^{*} \\
0,86^{*} \\
0,27^{* *} \\
0,62^{* *} \\
0,41^{* *} \\
0,38^{*}\end{array}$ \\
\hline $\begin{array}{l}\text { Técnica } \\
\text { Laparoscópica (\%) } \\
\text { Abierta (\%) }\end{array}$ & $\begin{array}{l}11(11,7) \\
83(88,3)\end{array}$ & $\begin{array}{r}1(3,6) \\
27(96,4)\end{array}$ & $\begin{array}{r}12(9,8) \\
110(90,2)\end{array}$ & 0.21 * \\
\hline $\begin{array}{l}\text { Invasión tumoralc } \\
\text { T3 (\%) } \\
\text { T4 (\%) }\end{array}$ & $\begin{array}{l}84(89,4) \\
10(10,6)\end{array}$ & $\begin{array}{r}27(96,4) \\
1(3,6)\end{array}$ & $\begin{array}{r}111(91) \\
11(9)\end{array}$ & $0,23^{* *}$ \\
\hline Adyuvancia (\%) & $20(21,3)$ & $6(21,4)$ & $26(21,3)$ & $0,99 *$ \\
\hline
\end{tabular}

SD, Desviación estándar; ASA, american society of anesthesiologists. ${ }^{a}$ Enfermedad o medicamentos inmunosupresivos concomi-

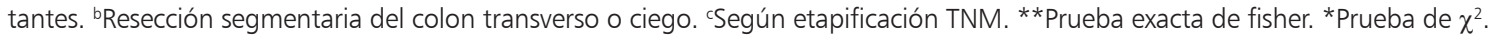

Tabla 2. Complicaciones perioperatorias en pacientes con NLR < a 5 y NLR $\geq$ a 5

\begin{tabular}{|c|c|c|c|c|}
\hline \multirow[b]{2}{*}{ Complicación } & \multicolumn{2}{|c|}{ NLR $<5$} & \multicolumn{2}{|c|}{ NLR $\geq 5$} \\
\hline & $\begin{array}{c}\text { Casos } \\
(n=20)\end{array}$ & $\%$ del Total & $\begin{array}{c}\text { Casos } \\
(n=15)\end{array}$ & $\%$ del Total \\
\hline Reintubación/Necesidad de ventilación mecánica & 1 & 5,0 & & \\
\hline Fiebre post operatoria & 1 & 5,0 & 2 & 13,3 \\
\hline íleo & 3 & 15,0 & 2 & 13,3 \\
\hline Fibrilación auricular & 1 & 5,0 & & \\
\hline Derrame pleural & 2 & 10,0 & 1 & 6,7 \\
\hline Retención urinaria & 1 & 5,0 & & \\
\hline Sepsis & 1 & 5,0 & 2 & 13,3 \\
\hline Infección sitio quirúrgico & 3 & 15,0 & 1 & 6,7 \\
\hline Dehiscencia herida & 1 & 5,0 & & \\
\hline Diarrea por C. difficile & 1 & 5,0 & & \\
\hline Filtración de anastomosis & 2 & 10,0 & 2 & 13,3 \\
\hline Crisis hipertensiva & 1 & 5,0 & & \\
\hline Absceso intraabdominal & & & 1 & 6,7 \\
\hline Insuficiencia renal aguda & & & 1 & 6,7 \\
\hline Neumonía & & & 1 & 6,7 \\
\hline Atelectasias & & & 1 & 6,7 \\
\hline Trombosis venosa profunda & & & 1 & 6,7 \\
\hline Hemorragia digestiva & 2 & 10,0 & & \\
\hline
\end{tabular}


Tabla 3. Análisis multivariado mediante regresión logística de factores pronósticos para complicaciones perioperatorias en pacientes con cáncer de colon etapa II

\begin{tabular}{|lccc|}
\hline & \multicolumn{3}{c|}{ Complicaciones } \\
& $\begin{array}{l}\text { Odds } \\
\text { ratio }\end{array}$ & $\begin{array}{c}\text { p } \\
\text { value }\end{array}$ & $\mathbf{9 5 \%}$ IC \\
NLR $\geq 5$ & 3,06 & 0,033 & $1,09-8,57$ \\
Edad & 1,01 & 0,635 & $0,97-1,05$ \\
\hline Sexo masculino & 1,44 & 0,440 & $0,57-3,64$ \\
\hline ASA 3-4 & 2,50 & 0,169 & $0,68-9,21$ \\
\hline Inmunosupresión & 1,64 & 0,458 & $0,44-6,09$ \\
\hline Adyuvancia & 0,51 & 0,366 & $0,12-2,18$ \\
\hline Técnica abierta & 0,39 & 0,179 & $0,09-1,55$ \\
\hline T4 ${ }^{\text {a }}$ & 1,42 & 0,691 & $0,25-7,98$ \\
\hline
\end{tabular}

asegún etapificación TNM.

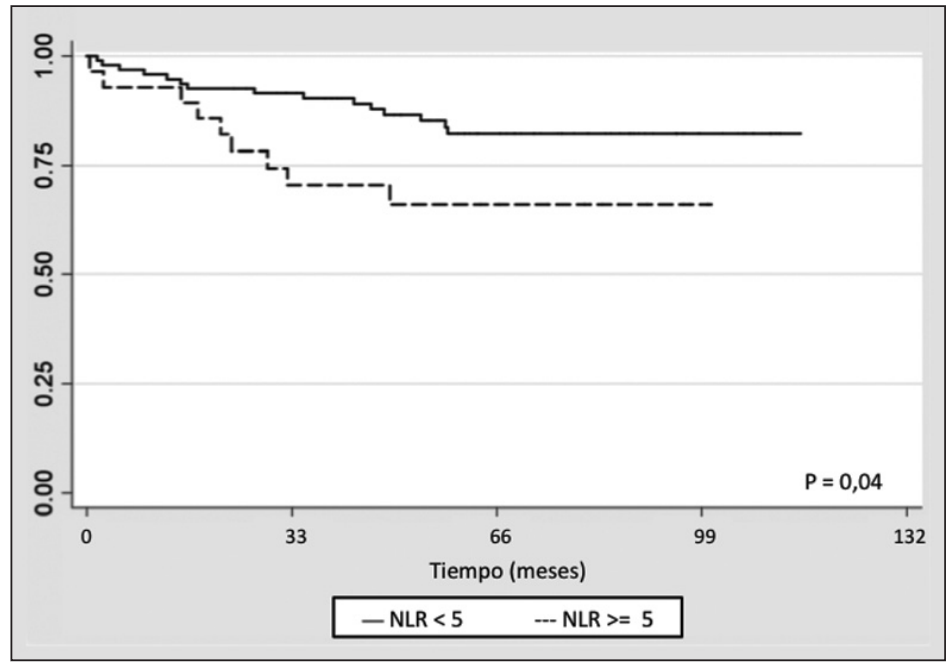

Figura 1. Curvas de Kaplan-Meier para sobrevida global en pacientes con cáncer de colon etapa II según NLR.

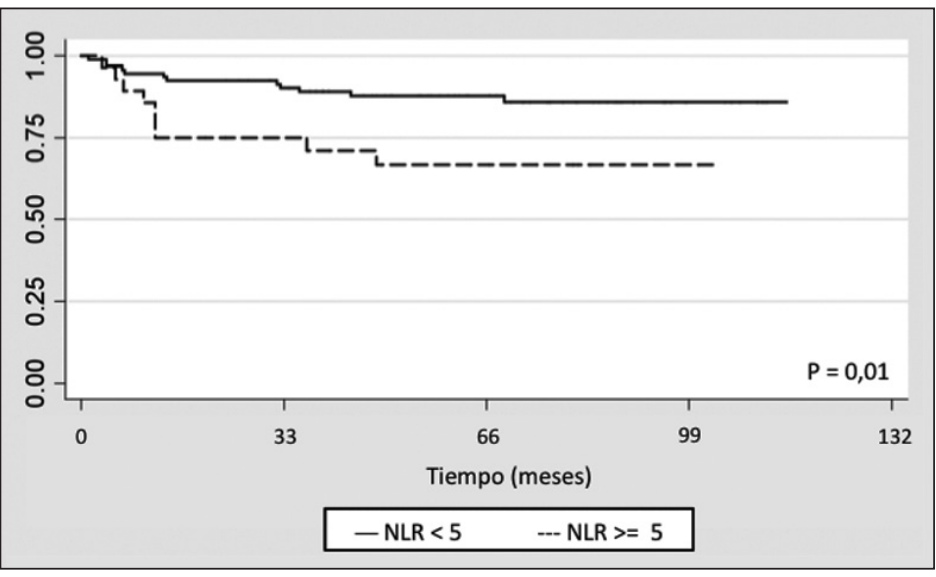

Figura 2. Curvas de Kaplan-Meier para sobrevida libre de enfermedad en pacientes con cáncer de colon etapa II según NLR. 


\section{Discusión}

Nuestros resultados muestran que, en pacientes sometidos a cirugía electiva para cáncer de colon en etapa II, el NLR es una herramienta de pronóstico independiente de otros factores de riesgo convencionales. Hemos encontrados 3 veces mayor riesgo de complicaciones perioperatorias, además de una menor sobrevida global y libre de enfermedad en el grupo con NLR elevado.

La asociación entre marcadores inflamatorios y patología oncológica maligna no es nueva. Virchow, en la mitad del siglo XIX, fue el primero en observar leucocitos en el tejido tumoral, y planteó la hipótesis que la inflamación tiene un rol importante en el desarrollo de tumores malignos ${ }^{18}$. Sin embargo, no fue hasta la presente década que se comenzó a entender el microambiente inflamatorio tumoral, y la respuesta inflamatoria del huésped inducida por el tejido tumoral. La relación entre un NLR elevado y malos resultados oncológicos no está completamente comprendida, sin embargo, se han postulado varios mecanismos posibles. Se cree que los neutrófilos son la fuente primaria de factor de crecimiento endotelial (VEGF), al cual se le ha atribuido un rol fundamental en la angiogénesis, proceso que aumenta la capacidad del tumor para diseminarse ${ }^{19,20}$. Dado lo anterior, parece coherente concluir que los pacientes con NLR elevado, es decir, que tienen una neutrofilia relativa, tienen mayor riesgo de recidiva. Por otro lado, está claro que la elevación de los marcadores inflamatorios sistémicos está asociado a linfocitopenia y una respuesta alterada de los linfocitos $\mathrm{T}$ hacia el tumor ${ }^{21,22}$. La respuesta inmune hacia los tumores está determinada por la inmunidad celular, la cual es dependiente de la población linfocitaria. Una linfocitopenia relativa podría afectar el pronóstico. En la misma línea, algunos estudios han demostrado que los pacientes con menos infiltrados linfocitarios en los márgenes tumorales tienen peor pronóstico ${ }^{23-26}$ y, según el sistema de etapificación de Jass ${ }^{27}$, un mayor infiltrado linfocitario en el tumor está asociado a una mejor chance de sobrevida. Otros estudios han validado el hecho que un tumor tenga mayor infiltrado inflamatorio, principalmente compuesto por linfocitos, se correlaciona con mejor pronóstico ${ }^{28,29}$. La integración de ambas hipótesis, neutrofilia y linfocitopenia, es la que probablemente le confiere al NLR la capacidad de identificar los pacientes en riesgo de complicación y pronóstico más ominoso.

Tal como lo mencionaron Walsh et al previamente, el NLR se puede calcular de los exámenes preoperatorios de rutina, tiene una sensibilidad y especificidad razonable y su uso no requiere recursos adicionales ${ }^{17}$. Sin embargo, el efecto independiente de la neutrofilia y la linfocitopenia y sus resultados aún está por determinarse.

Varios factores podrían resultar en una respuesta inflamatoria sistémica preoperatoria, incluyendo infección clínicamente evidente, condiciones crónicas que determinen inflamación, así como cirugía de urgencia. Aún cuando los pacientes con estas condiciones fueron excluidos del estudio, no podemos descartar la existencia de condiciones subclínicas, ya sean infecciosas o inflamatorias que podrían afectar el NLR.

Pese a que no observamos asociación estadísticamente significativa entre la distribución de tumores T4 y el NLR, el compromiso transmural podría ser un gatillante potente de la respuesta inflamatoria, que a su vez podría elevar el NLR. Por otro lado, la distribución de pacientes según la clasificación ASA no fue igual en ambos grupos de NLR, esto podría afectar el efecto del NLR en las complicaciones post operatorias, sin embargo, cuando se realizó el análisis multivariado, la asociación del ASA 3-4 con complicaciones post operatorias se perdió en presencia del NLR. Este hallazgo sugiere que la asociación entre un ASA elevado y las complicaciones postoperatorias son explicadas por el NLR $\geq 5 \mathrm{u}$ otras variables que se ven indirectamente representadas en el NLR. Por lo tanto, el NLR parece ser un factor predictor independiente de complicaciones postoperatorias.

Luego de su resección quirúrgica, entre 30 a $40 \%$ de los carcinomas de colon son diagnosticados como estadio II según la clasificación de la AJCC $^{30,31}$. La habilidad para identificar los subgrupos con alto riesgo de recurrencia podría mejorar las estrategias del tratamiento, por ejemplo asociando la quimioterapia adyuvante. Se han identificado varios marcadores histopatológicos de mal pronóstico en etapa II y se usan como indicación de quimioterapia adyuvante ${ }^{32}$, sin embargo, sólo una fracción de estos pacientes efectivamente recibe el tratamiento, como fue reportado por Morris et $a^{33}$. La mayoría de los marcadores explorados, tales como la ploidía de $\mathrm{ADN}$, marcadores moleculares y micrometástasis, 
no han tenido un impacto significativo en modificaciones del tratamiento ${ }^{34-36}$.

Nuestro estudio, dado su diseño, posee algunas limitaciones. La naturaleza observacional afecta las conclusiones de causalidad y resulta imposible excluir confundentes no medidos. Como ya hemos mencionado anteriormente, observamos que la mayoría de los confundentes incluidos en el modelo logístico no resultaron en una asociación significativa con la complicaciones post operatorias, sin embargo, esto puede estar explicado por el bajo número de eventos observados. Por otro lado, algunas fortalezas de este trabajo deben mencionarse. Primero, la información y la pérdida de pacientes en el seguimiento fueron controlados al máximo hasta el cierre del seguimiento en el 2007. Segundo, el hecho de que sea un centro único participante contribuye a un proceso más uniforme de obtención de la información. Tercero, los resultados observados en este estudio son consistentes con otras series similares ${ }^{37,38}$. Finalmente, los criterios de selección se han explicitado claramente para que el lector se forme un juicio sobre la validez externa de nuestros resultados.

En conclusión, hemos observado que un NLR elevado se asocia a complicaciones postoperatorias y peor sobrevida global y libre de enfermedad.

\section{Referencias}

1. Jemal A, Siegel R, Ward E, Hao Y, Xu J, Murray T, et al. Cancer statistics, 2008. CA Cancer J Clin 2008; 58 (2): 71-96.

2. Cancerstats 2004. Cancer research UK. Available at: http://info.cancerresearchuk.org/cancerstats/types/ bowel/. Accessed July 30, 2009.

3. McArdle CS, Hole DJ. Outcome following surgery for colorectal cancer: analysis by hospital after adjustment for case-mixanddeprivation. Br J Cancer 2002; 86: 331-5.

4. NIH consensus conference. Adjuvant therapy for patients with colon and rectal cancer. JAMA 1990; 264: 1444-50.

5. Association of Coloproctology of Great Britain and Ireland. Guidelines for the management of colorectal cancer. London (UK): Association of Coloproctology of Great Britain and Ireland; 2007. Available at: www.guideline.gov/summary/summary.aspx?doc_id=11331\#s23. Accessed July 30, 2009.

6. National Health and Medical Research Council of Australia. Guidelines for the Prevention, Early Detection and Management of Colorectal Cancer (CRC). National
Health and Medical Research Council of Australia: Canberra, 2005. Available at: www.nhmrc.gov.au/guidelines/ health_guidelines.htm. Accessed July 30, 2009.

7. Morris MJ, Platell C, De Boer B, McCaul K, Lacopetta B. Population-based study of prognostic factors in stage II colon cancer. Br J Surg 2006; 93 (7): 866-71.

8. Graziano F, Cascinu S. Prognostic molecular markers for planning adjuvant chemotherapytrials in Dukes' B colorectal cancer patients: how much evidence is enough? Ann Oncol 2003; 14: 1026-38.

9. Balkwill F, Mantovani A. Inflammation and cancer: back to Virchow? Lancet 2001; 357: 539-545.

10. Coussens LM, Werb Z. Inflammation and cancer. Nature 2002; 420: 860-7.

11. Jaiswal M, LaRusso NF, Burgart LJ, Gores GJ. Inflammatory cytokines induce DNA damage and inhibit DNA repair in cholangiocarcinoma cells by a nitricoxidedependent mechanism. Cancer Res 2000; 60: 184-90.

12. Jackson JR, Seed MP, Kircher CH, Willoughby DA, Winkler JD. The codependence of angiogenesis and chronic inflammation. FASEB J. 1997; 11: 457-65.

13. Zahorec R. Ratio ofneutrophil to lymphocyte count is a rapid and simple parameter of systemic inflammation and stress in critically ill. Bratisl Lek Listy 2001; 102: 5-14.

14. Duffy BK, Gurm HS, Rajagopal V, Gupta R, Ellis SG, Bhatt DL. Usefulness of an elevated neutrophil to lymphocyte ratio in predicting long term mortality after percutaneous coronary intervention. Am J Cardiol 2006; 97 (7): 993-6.

15. Halazun KJ, Aldoori A, Malik HZ, Al-Mukhtar A, Prasaid KR, Toogood GJ, et al. Elevated preoperative neutrophil to lymphocyte ratio predicts survival following hepatic resection for colorectal liver metastases. Eur J Surg Oncol 2008; 34: 55-60.

16. Sarraf KM, Belcher E, Raevsky E, Nicholson AG, Goldstraw P Lim E. Neutrophil/lymphocyte ratio and its association with survival after complete resection in non-small cell lung cancer. J Thorac Cardiovasc Surg. 2009; 137 (2): 425-8.

17. Walsh SR, Cook EJ, Goulder F, Justin TA Keeling NJ. Neutrophil-lymphocyte ratio as a prognostic factor in colorectal cancer. J Surg Oncol 2005; 91 (3): 181-4.

18. Balkwill F, Mantovani A. Inflammation and cancer: back to Virchow? Lancet 2001; 357: 539-45.

19. Kusumanto YH, Dam WA, Hospers GA, Sluiter WJ, Dam WA, Meijer C, et al. Platelets and granulocytes, in particular the neutrophils, form important compartments for circulating vascular endothelial growth factor. Angiogenesis 2003; 6: 283-7.

20. Tanigawa N, Amaya N, Matsumura M, Shimomatsuya T. 
Índice neutrófilo/linfocito como pronóstico en cáncer de colon - G. I. Urrejola et al

Correlation between expression of vascular endothelial growth factor and tumor vascularity, and patient outcome in human gastric carcinoma. J ClinOncol 1997; 15: 826-2.

21. Du Klos TW, Mold C. C-reactive protein: an activator of innate immunity and a modulator of adaptive immunity. Immunol Res 2004; 30: 261-77.

22. Nozoe T, Matsumata T, Sugimachi K. Preoperative elevation of serum C-reactive protein is related to impaired immunity in patients with colorectal cancer. Am J Clin Oncol 2000; 23: 263-6.

23. Ropponen KM, Eskelinen MJ, Lipponen PK, Alhava E, Kosma VM. Prognostic value of tumor-infiltrating lymphocytes (TILs) in colorectal cancer. J Pathol 1997; 182: 318-29.

24. Jass JR. Lymphocytic infiltration and survival in rectal cancer. J Clin Pathol 1986; 39: 585-9.

25. Svennevig JL, Lunde OC, Holter J, Bjorsvik D. Lymphoid infiltration and prognosis in colorectal carcinoma. $\mathrm{Br} \mathrm{J}$ Cancer 1984; 49: 375-7.

26. Okano K, Maeba T, Moroguchi A, Ishimura K, Karasawa $\mathrm{Y}$, Izuishi $\mathrm{K}$ et al. Lymphocytic infiltration surrounding liver metastases from colorectal cancer. J Surg Oncol 2003; 82 (1): 28-33.

27. Jass JR, Love SB, Northover JM. A new prognostic classification of rectal cancer. Lancet 1987; 1 (8545): 1303-6.

28. Ropponen KM, Eskelinen MJ, Lipponen PK, Alhava E, Kosma VM. Prognostic value of tumour-infiltrating lymphocytes (TILs) in colorectal cancer. J Pathol 1997; 182: 318-24.

29. Canna K, McArdle PA, McMillan DC, McNIcol AM, Smith GW, McKee RF, et al. The relationship between tumour T-lymphocyte infiltration, the systemic inflammatory response and survival in patients undergoing curative resection for colorectal cancer. $\mathrm{Br} \mathrm{J}$ Cancer 2005; 92 (4): 651-4.

30. Jemal A, Tiwari RC, Murray T Ghafoor A, Samuels A,
Ward A, et al. American Cancer Society. Cancer statistics 2004. CA Cancer J Clin 2004; 54: 8-29.

31. Jessup JM, McGinnis LS, Steele GD Jr, Menk HR, Winchester DP. The National Cancer Data Base. Report on colon cancer. Cancer 1996; 78: 918-26.

32. Graziano F, Cascinu S. Prognostic molecular markers for planning adjuvant chemotherapy trials in Dukes' B colorectal cancer patients: how much evidence is enough? Ann Oncol 2003; 14: 1026-38.

33. Morris M, Platell C, McCaul K, Millward M, van Hazel $\mathrm{G}$, Bayliss E, et al. Survival rates for stage II colon cancer patients treated with or without chemotherapy in a population-based setting. Int J Colorectal Dis 2007; 22: 887-95.

34. Shinji S, Naito Z, Ishiwata T, Tanaka N, Furukawa K, Suzuki H, et al. Clinical and Biological Significance of Lymph Node Micrometastasis in Colorectal Cancer. J Nippon Med Sch 2007; 74: 77-80.

35. Santagostino A, Saggia C, Migliora P, Pavanelli MC, Forti G, Biaggi G, et al. Prospective study on prognostic significance of DNA ploidy and Ki-67 expression in colorectal cancer. J Biol Regul Homeost Agents 2007; 21 (1-2): 13-20

36. Buglioni S, D’Agnano I, Cosimelli M, Vaselli S, DeAngelo C, Tedesco M, et al. Evaluation of multiple bio-pathological factors in colorectal adenocarcinomas: independent prognostic role of p53 and bcl-2. Int J Cancer 1999; 84 (6): 545-52.

37. Ding PR, An X, Zhang RX, Fang YJ, Li LR, Chen G, et al. Elevated preoperative neutrophil to lymphocyte ratio predicts risk of recurrence following curative resection for stage IIA colon cancer. Int J Colorectal Dis 2010; 25 (12): 1427-33.

38. Hung HY, Chen JS, Yeh CY, Changchien CR, Tang R, Hsieh PS, et al. Effect of preoperative neutrophil-lymphocyte ratio on the surgical outcomes of stage II colon cancer patients who do not receive adjuvant chemotherapy. Int J Colorectal Dis 2011; 26 (8): 1059-65. 